\title{
ReMed: rapport annuel 2018
}

ReMed, Comité de direction

\section{Connaissez-vous ReMed, le réseau de soutien pour les médecins?}

ReMed est un réseau de soutien destiné aux médecins introduit dans toute la Suisse en 2010. Il transmet aux médecins ses connaissances et ses expériences en faveur de la promotion de la santé et de la prévention afin de les sensibiliser à leur propre santé. ReMed dispose d'un large éventail de prestations pour épauler les médecins en situation de crise. Son objectif est de maintenir les médecins en bonne santé et de préserver leur capacité fonctionnelle afin de garantir la sécurité des patients et une qualité élevée des soins à la population.

\section{L'offre principale de ReMed: première intervention et mise en réseau}

Pour ReMed, la santé des médecins est essentielle et le réseau accorde une attention particulière aux risques spécifiques du métier et à la prévention. Chez ReMed, le statut d'un médecin qui demande de l'aide est identique à celui d'un patient, même si cela ne veut pas dire que le médecin concerné est malade.

Pendant toute la durée du processus, ReMed cherche à mettre en réseau les offres déjà existantes et collabore avec tous les professionnels qui partagent ses objectifs. Après le premier contact, ReMed répond dans les 72 heures au médecin qui sollicite de l'aide et essaie de comprendre la situation et de trouver des solutions.

La grande majorité des demandes de soutien adressées à ReMed permettent de proposer des solutions et si nécessaire de mettre les médecins en relation avec des médecins de famille, des spécialistes (p. ex. psychiatrespsychothérapeutes) ou d'autres professionnels (p. ex. avocats, fiduciaires)

\section{3 demandes de soutien en 2018}

Par rapport à l'année précédente, le nombre de demandes a légèrement augmenté pour atteindre 143. La plupart des prises de contact ont eu lieu durant le mois de novembre. Cette augmentation constante montre clairement la nécessité d'une telle offre de soutien. Les médecins du Comité de direction ont répondu à 114 demandes de Suisse alémanique, 28 de Suisse romande et une du Tessin, parmi lesquelles $70 \%$ concernaient des femmes et 30\% des hommes, exerçant pour la majorité dans le secteur hospitalier. Le stress au travail, le burn-out et la dépression sont les principales causes évoquées par les personnes qui font appel à ReMed (cf. fig. 1).

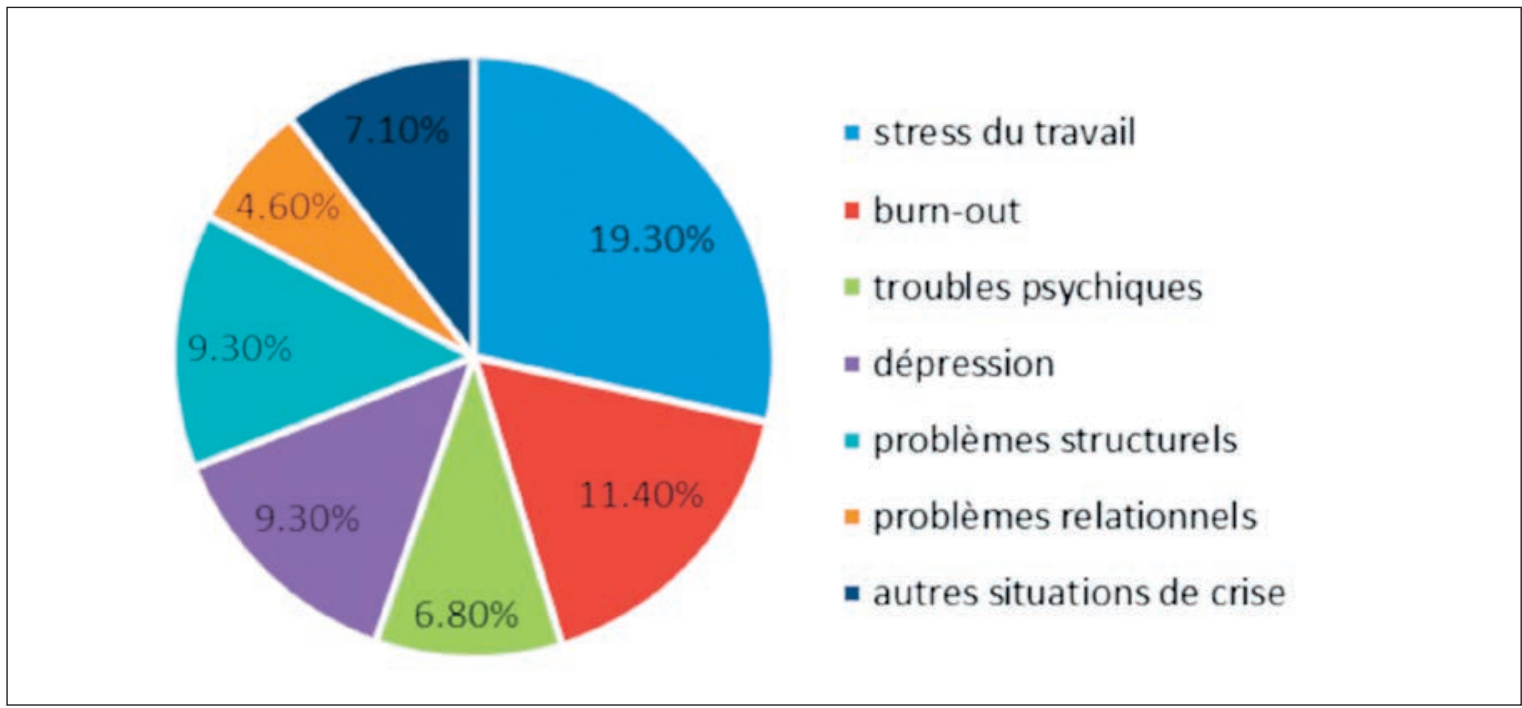

Figure 1: Raisons incitant à contacter ReMed (plusieurs réponses possibles). 


\section{Mise en réseau}

Le stress au travail, le burn-out et la double charge de la maternité et du travail sont les raisons les plus fréquemment citées par les personnes faisant appel à ReMed. L'Association suisse des médecins-assistant(e)s et chef(fe)s de clinique (ASMAC) a également constaté une augmentation des questions liées à la double charge que constituent le travail et la famille, à l'égalité et à la conciliation entre carrière et travail à temps partiel. En 2018, ReMed et l'ASMAC ont pris ces questions au sérieux et convenu de les aborder ensemble afin de trouver des solutions communes. En 2018, le réseau de ReMed a été une nouvelle fois étendu et renforcé par de nouveaux premiers répondants. D'importants liens existent avec des organisations en Suisse alémanique et en Suisse romande dans le but d'optimiser et d'élargir constamment l'offre; des groupes de parole verront par exemple le jour prochainement dans des hôpitaux romands.

\section{Nouveaux groupes de coaching à Saint-Gall}

A la fin 2018, un groupe de coaching a démarré à SaintGall. De tels groupes de parole existent depuis 2016 à Berne et Zurich. Animés par un expert, ils offrent un soutien aux médecins qui doivent faire face aux sollicitations et défis liés à leur activité professionnelle. Les conflits et les difficultés au travail sont abordés et les solutions discutées et élaborées ensemble. Vous trouverez des informations sur les différents groupes sur

Internet (www.swiss-remed.ch).

\section{Assurer notre visibilité}

Le réseau de soutien continue de faire connaître son offre au travers d'ateliers et de représentations théâtrales lors de congrès et de sessions de formation et dans les institutions. Nous avons ainsi pu faire connaître notre offre de manière différenciée à un cercle toujours plus large de personnes. Ces interventions ont contribué à lever toujours plus le tabou sur les sollicitations professionnelles auxquelles les médecins sont confrontés. En 2018, ReMed a également assuré sa présence dans les médias par la publication d'articles. Les témoignages publiés ces dernières années dans le Bulletin des médecins suisses ont rencontré un grand intérêt et un écho positif auprès des lecteurs et montrent comment ReMed est venu en aide aux personnes concernées.

\section{Témoignages et documentation}

Sur le site www.swiss-remed.ch, vous découvrirez des témoignages passionnants, de la documentation et de précieuses informations concernant le réseau de soutien.

Le programme national ReMed avec le Comité de direction et le réseau d'intervenants sera aussi là en 2019 pour les médecins en situation de crise. Les médecins qui souhaitent recevoir des conseils ou une aide peuvent prendre contact $24 \mathrm{~h}$ sur 24 via la ligne d'assistance (0800 0 73633) ou par courriel (help[at]swissremed.ch).
ReMed

Secrétariat

Case postale 55

CH-3000 Berne 15 